\title{
Design and control of a lower limb exoskeleton for robot-assisted gait training
}

\author{
Pieter Beyl*, Michaël Van Damme, Ronald Van Ham, Bram Vanderborght and Dirk Lefeber \\ Department of Mechanical Engineering, Vrije Universiteit Brussel, Brussels, Belgium
}

(Received 30 September 2008; final version received 23 January 2009)

\begin{abstract}
Robot-assisted rehabilitation of gait still faces many challenges, one of which is improving physical human-robot interaction. The use of pleated pneumatic artificial muscles to power a step rehabilitation robot has the potential to meet this challenge. This paper reports on the development of a gait rehabilitation exoskeleton with a knee joint powered by pleated pneumatic artificial muscles. It is intended as a platform for the evaluation of design and control concepts in view of improved physical human-robot interaction. The design was focused on the optimal dimensioning of the actuator configuration. Safety being the most important prerequisite, a proxy-based sliding mode controller (PSMC) was implemented as it combines accurate tracking during normal operation with a smooth, slow and safe recovery from large position errors. Treadmill walking experiments of a healthy subject wearing the powered exoskeleton show the potential of PSMC as a safe robot-in-charge control strategy for robot-assisted gait training.
\end{abstract}

Keywords: robot-assisted gait rehabilitation; powered exoskeleton; compliant actuator; proxy-based sliding mode control

\section{Introduction}

Locomotion training is considered an effective approach to helping incomplete spinal cord injured (SCI) subjects recover their walking capabilities (Wernig and Muller 1992; Wirz et al. 2001). The automation of locomotion training by means of a powered exoskeleton potentially improves training efficacy and rehabilitation outcome (Krebs et al. 2000; Colombo et al. 2001). Physiological gait patterns can be produced and adapted with higher accuracy. Automatic monitoring of the patient's progress allows the development of more adequate training schemes. The robotic device relieves strain on therapists, thus enabling longer training sessions and allowing the same number of therapists to supervise more patients.

Several research groups are developing powered exoskeletons for the rehabilitation of gait. The Lokomat (Hocoma AG, Switzerland) is one of the few commercialised step rehabilitation systems and well reported in literature (Colombo et al. 2000; Jezernik et al. 2003). It consists of a treadmill, an overhead suspension system with a harness and a robotic exoskeleton powered by electric drives at the hips and knees that assists the patient in performing walking movements on the treadmill. The Lokomat has undergone substantial testing with mobility impaired patients (Wirz et al. 2005). The Biomechatronics Lab of the University of California has developed several robotic devices (ARTHuR: ambulation-assisting robotic tool for human rehabilitation, PAM: pelvic assist manipulator, POGO: pneumatically operated gait orthosis) to study the mechanisms of motor learning and the effects of robot-assisted gait training in SCI patients (Reinkensmeyer et al. 2006). The rationale behind these devices is to assist only as needed and provide a sensory input as natural as possible to maximise therapy outcome. The LOwer extremity Powered ExoSkeleton (LOPES) project of the Laboratory of Biomechanical Engineering at the University of Twente aims at improving gait training for post-stroke patients. Their robotic exoskeleton uses series elastic and Bowden cable-based actuators to assist in flexion/extension at the knee and both flexion/extension and adduction/abduction at the hip (Veneman et al. 2007). Focus is on selective support of gait functions as opposed to continuous robotic assistance (Ekkelenkamp et al. 2007). At the University of Delaware (Agrawal et al. 2007) several unmotorised devices (GBO, SUE) and one motorised leg exoskeleton (ALEX) have been developed for gait assistance and training of the motor impaired. The Human Neuromechanics Laboratory of the University of Michigan focuses on lower limb orthoses for task-specific gait rehabilitation, powered by McKibben type pneumatic artificial muscles (Ferris et al. 2005). A complete lowerbody exoskeleton powered by the same type of actuators has been constructed at the Center for Robotics and Automation of the University of Salford (Costa and Caldwell 2006). From a broader perspective, research on assistive technology for the lower limbs needs to be mentioned. The latter comprise powered orthoses to compensate for gait insufficiency as well as robotic exoskeletons acting as a force/power augmenting device. Although these devices

*Corresponding author. Email: pbeyl@vub.ac.be 
are not intended to be used as gait rehabilitation aids, the ongoing related research inspires new developments in step rehabilitation robotics. An overview of the state-of-the-art can be found in Dollar and Herr 2008.

Although the clinical results of automated treadmill training reported in literature (Wirz et al. 2005) are encouraging, existing gait rehabilitation devices do suffer from a few limitations and future research faces some important challenges. First of all, most devices do not actively support the ankle joint, which results in poorly constrained and unnatural motion of the foot. The lack of actuation at the ankle possibly has an adverse effect on the recovery of normal gait, as in normal human walking the largest peak moments of force occur at the ankle joint, just before toe off. Also some causes of gait dysfunction like dropped foot or spastic foot extension cannot be adequately countered during therapy. Secondly, the use of a suspension system with a harness leads to an unnatural body weight distribution and hinders balance and postural reactions of the patient, required in functional walking. Thirdly, there is a need for more physiological robotic joints allowing movements closer to the three-dimensional kinematics of functional human gait. Fourthly, due to their actuation system and control approach these robots often lack compliance, which is considered an important prerequisite for safe human-robot interaction (Bicchi and Tonietti 2004). There is a growing tendency towards patient-cooperative control strategies, where, instead of predefined movements being imposed, the patient's intentions and efforts are taken into account (Riener et al. 2005; Ekkelenkamp et al. 2007; Emken et al. 2008). Still, in many cases, either compliant behaviour is only built-in on the control level or only partially built-in on the hardware level. To date, although gradually drawing more attention, these four challenges have not yet been addressed in an integrated fashion.

We hypothesise that a device providing active ankle assistance, full body weight support, three-dimensional gait assistance and adaptable compliant behaviour, that is both built-in in the actuation system and taken advantage of on the control level, has the potential to improve the quality of robot-assisted gait training. Our goal is to develop such a device and to meet the aforementioned challenges by means of a special type of actuator: the pleated pneumatic artificial muscle (PPAM) (Daerden and Lefeber 2001, 2002; Verrelst et al. 2006). This actuator is inherently compliant and we believe that, in conjunction with adequate control strategies, it will provide the necessary safe and soft touch for a better human-robot interaction. Additionally, the actuator's high pulling force enables the robot to carry the full weight of the patient, without resorting to an overhead suspension system. Before starting the development of the complete gait rehabilitation robot, however, we are investigating aspects of design and control on a smaller and less complex scale. Therefore the first phase of the project involves the development of a proof-of-concept unilateral leg exoskeleton with a PPAM powered knee joint.

In this paper we study the design and control of this powered knee exoskeleton. Both design and control of robotic applications powered by pneumatic artificial muscles are very challenging. To compensate for the limited contraction range and non-linear output of the pneumatic muscles a four bar linkage mechanism has been conceived to increase the range of motion and to shape the torque characteristic of the actuated knee joint, while keeping the design fairly compact. The intrinsic safety of the system due to actuator compliance was preserved as much as possible by selecting an adequate controller. A proxy-based sliding mode controller (PSMC) has been implemented, since it combines good tracking performance with a safe response to large deviations from the target position. The performance of the controller has been tested in actual gait tracking experiments. Initial treadmill walking experiments with a healthy subject are presented.

The paper is organised as follows. Section 2 describes the design of the prototype with an emphasis on the dimensioning of the actuation system. In Section 3 the control strategy is introduced and controller characteristics are discussed on the basis of simulation results. The experimental setup and results of initial walking experiments are presented and discussed in Section 4. In the final section we come to conclusions.

\section{Exoskeleton design}

\subsection{Mechanical structure}

The exoskeleton (see Figure 1) is unilateral and consists of an adjustable upper body link, upper leg link and lower leg

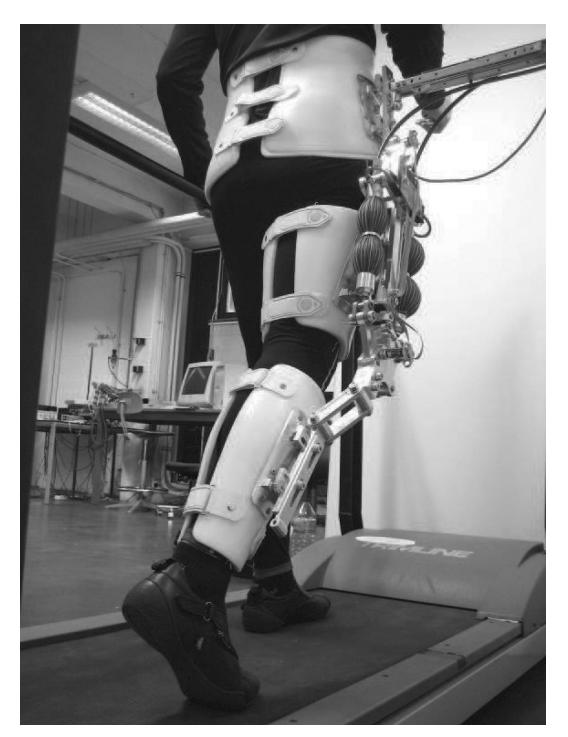

Figure 1. Close up of the unilateral exoskeleton with the knee joint powered by pleated pneumatic artificial muscles. 
link interconnected by hinge joints. Only the knee joint is powered by pleated pneumatic artificial muscles (PPAMs). The actuation system has been designed to provide the exoskeleton with enough power to fully support normal human gait in the absence of human knee joint power. The design procedure is explained in detail in Subsection 2.2. The actuators are tethered to an external pressurised air supply system with a high performance pressure regulating valve (Kolvenbach KPS 3/4-00) for each PPAM. As mentioned in the introductory section, the exoskeleton is intended as a proof-of-concept device for the development and testing of design and control concepts. For this reason, the design has been tailored to the requirements of an average male subject. For the adjustability of the device only small inter-subject variations have been taken into account. Two-piece rigid thermoplastic shells with a foam inlay and Velcro straps provide an adaptable fit at the lower and upper leg and the hip. Their position and orientation with respect to the exoskeleton frame are adjustable by means of slider mechanisms. Mechanical limiters prevent hyperextension and hyperflexion of the knee. A supportive arm (see Figure 2) with zero free length spring mechanism (Rahman et al. 1995) passively gravity-balances the exoskeleton to reduce asymmetrical loading of the subject wearing the device. The telescopic arm is connected to the upper body link by means of a ball joint. Additionally, the arm can pivot about a horizontal and vertical axis near the supporting wall. In this way all 6 degrees of freedom (DOF) of the upper body link are unconstrained, allowing unhindered treadmill walking. The weight of the exoskeleton, including the attachments, is $5.8 \mathrm{~kg}$.

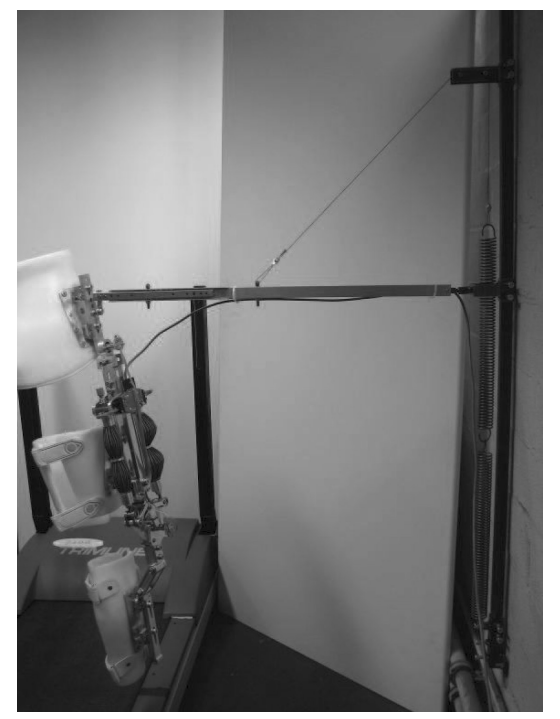

Figure 2. Unilateral exoskeleton with supportive arm.

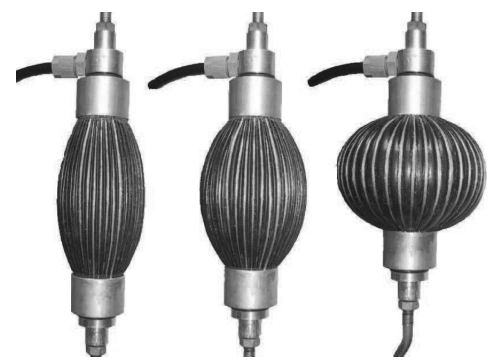

Figure 3. Pleated pneumatic artificial muscle at three different contraction levels.

\subsection{Actuation system}

\subsubsection{Pleated pneumatic artificial muscle}

The pleated pneumatic artificial muscle (PPAM, see Figure 3) is a lightweight, air-powered actuator that generates linear motion (Daerden and Lefeber 2001, 2002; Verrelst et al. 2006). Its core element is a reinforced pleated membrane that expands radially and contracts axially when pressurised, while exerting a pulling force in the longitudinal direction. The rationale behind the development of this type of PAM was to obtain improved characteristics with respect to the relatively well known McKibben muscle (Schulte, 1961). The latter shows substantial hysteresis, a high threshold pressure and a contraction range limited to $20-30 \%$. Due to its different working principle, the PPAM has reduced hysteresis, virtually no threshold pressure and a contraction range up to $40 \%$ depending on the muscle dimensions.

A mathematical model that describes shape, volume, diameter, exerted force and maximal contraction of PPAMs can be found in Daerden and Lefeber (2001), and Verrelst et al. (2006). Under the assumption of negligible membrane elasticity the force exerted by the muscle is given by

$$
F=p l_{0}^{2} f_{t 0}\left(\epsilon, l_{0} / R\right)
$$

In this expression, $p$ is the applied gauge pressure, $l_{0}$ is the muscle's maximal length, $R$ is its minimal radius and $\epsilon$ is the muscle contraction. Using $l$ for the actual muscle length, we have $\epsilon=1-l / l_{0} . f_{t 0}$ is a non-linear, dimensionless function that depends on contraction and on the design parameter $l_{0} / R$, called slenderness. This results in muscle-like behaviour, with high forces being generated at low contractions and low forces at high contractions. Figure 4 shows the varying force-contraction relation at different gauge pressures for a muscle with slenderness $l_{0} / R=8$ and $l_{0}=0.1 \mathrm{~m}$. To avoid excessive material loading, contraction should be kept above $5 \%$.

\subsubsection{Actuator configuration}

The PPAM is a single-acting actuator. To obtain a bidirectional rotational actuator, one has to configure two 


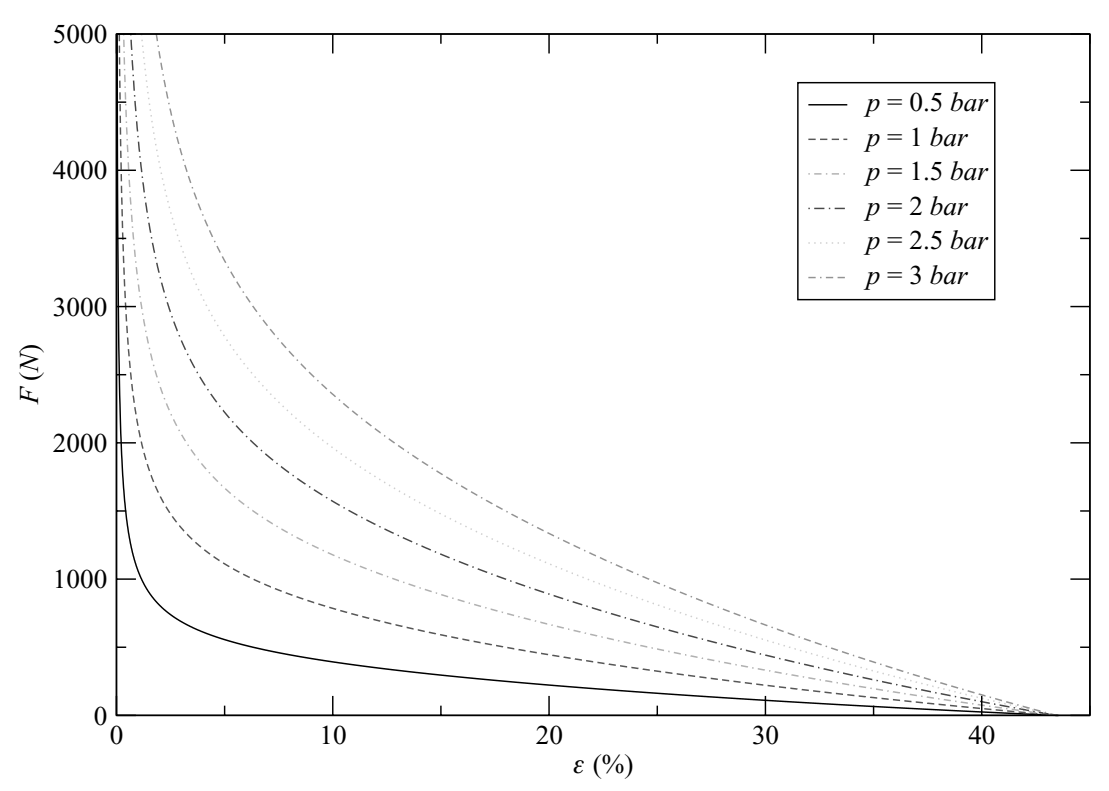

Figure 4. Force-contraction relation at different gauge pressures for a PPAM with $l_{0} / R=8$ and $l_{0}=0.1 \mathrm{~m}$.

single-acting actuators in an antagonistic configuration. The actuator forces can be transferred to the joint in different ways. A pulley-and-belt system for instance has a constant lever arm length, which favours compactness, but leaves the non-linear force-contraction characteristic of the PPAM uncompensated. A direct connection through fixed levers results in a variable lever arm length. This enables the torque-angle characteristic of the joint to be shaped in a more suitable way, but introduces spatial constraints, especially when a large angular range of motion is required. We have chosen a configuration with a four bar linkage mechanism at either side of the joint, as it combines the advantages of both of the aforementioned configurations, namely compactness and good torque matching.

Figure 5 depicts a schematic drawing of this configuration. The upper and lower link are interconnected by a hinge joint in $O$. Their relative angle is denoted by $q$. The extensor PPAMs and flexor PPAMs are connected to the upper link through hinging points $B_{i}$ and to the lower link through four bar linkages with hinging points $O, F_{i}, D_{i}, G_{i}$, with $i=1,2$ denoting the extensor and flexor side, respectively. The joint kinematics are defined by linkage parameters $f_{i}$, $d_{i}, g_{i}, c_{i}$, lengths $b_{i}, l_{i}$ and angles $\alpha_{i}$. The characteristics of the PPAMs are determined by $l_{0 i}$ and $R_{i}$, the single muscle's maximal length and minimal radius (see Subsection 2.2.1), $s_{i}$ and $p_{i}$, the number of single muscles in series and parallel, and contraction level $\epsilon_{0 i}$ at a certain angle, in this case $q=0^{\circ}$. In summary, the characteristics of this particular configuration are fully defined by the following two sets of 12 mechanical design parameters each,

$$
X_{i}=\left[l_{0 i}, R_{i}, s_{i}, p_{i}, \epsilon_{0 i}, b_{i}, l_{i}, f_{i}, d_{i}, c_{i}, g_{i}, \alpha_{i}\right]
$$

The optimisation procedure involved in selecting the design parameters enumerated in Equation 2 is explained in the next section. Once these parameters are determined by design, the characteristics of the powered joint are solely dependent on the joint angle $q$ and the extensor and flexor muscle pressures $p_{1}$ and $p_{2}$ respectively. Taken into account the kinematics of the configuration defined by Equation 2 and the force-contraction relation of the PPAM in Equation 1 , the torque generated by the PPAMs at the joint can be formulated as

$$
\tau=p_{1} m_{1}(q)+p_{2} m_{2}(q)
$$

with $m_{i}(q)$ torque functions determined by the design parameters and $p_{i}$ the muscle gauge pressures. Due to air compressibility the joint has intrinsic compliance. The stiffness (inverse of compliance) of the joint can be formulated, using Equation 3, as

$$
\begin{aligned}
K & =-\frac{d \tau}{d q} \\
& =-\frac{d p_{1}}{d q} m_{1}-p_{1} \frac{d m_{1}}{d q}-\frac{d p_{2}}{d q} m_{2}-p_{2} \frac{d m_{2}}{d q}
\end{aligned}
$$

The terms $d p_{i} / d q$ can be approximated (Vanderborght et al., 2006) by

$$
\frac{d p_{i}}{d q}=-n\left(p_{i}+P_{\mathrm{atm}}\right) \frac{1}{V_{i}(q)} \frac{d V_{i}(q)}{d q}
$$

with $V_{i}(q)$ the muscle volume, $P_{\text {atm }}$ the atmospheric pressure and $n$ the polytropic coefficient. Equation (5) has been 


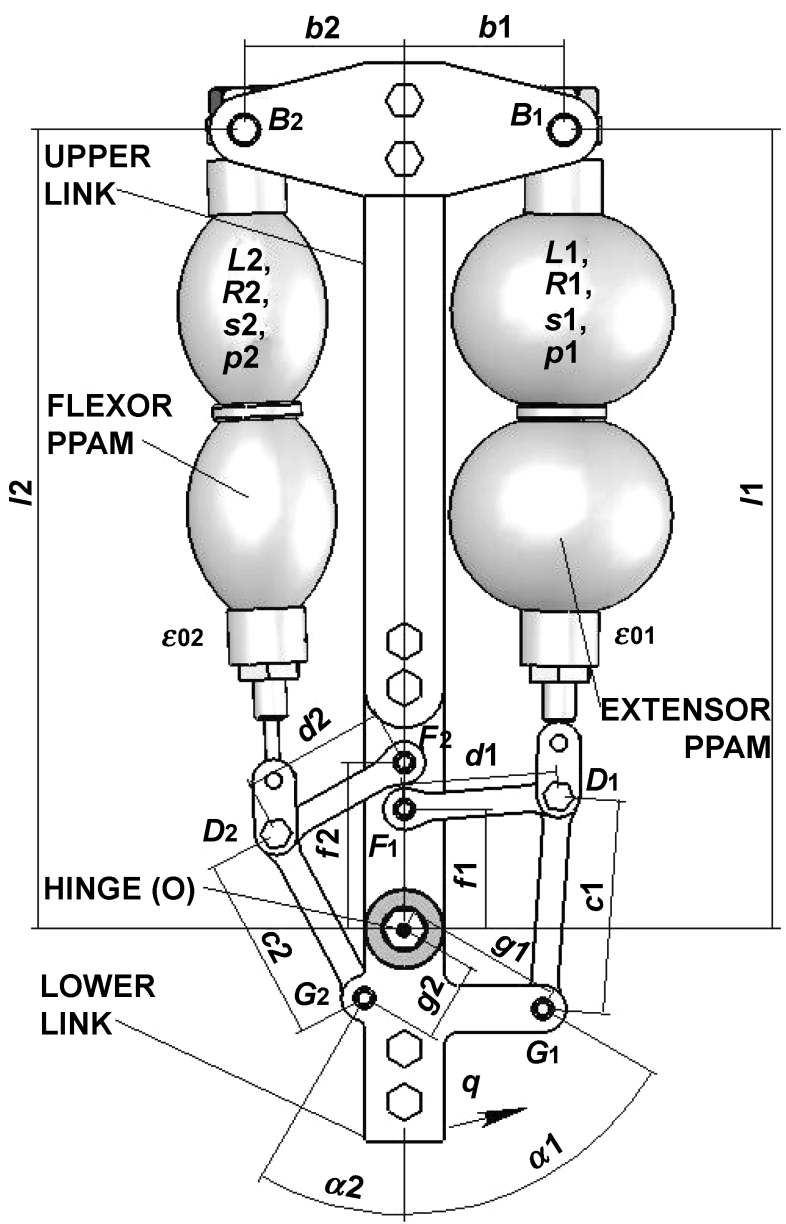

Figure 5. Schematic drawing and determining mechanical parameters of an antagonistic configuration of pleated pneumatic artificial muscles with four bar linkages.

derived from the polytropic law, linking pressure variations inside the muscle to muscle volume variations. The muscle volume $V_{i}(q)$ can be found in Verrelst et al. 2006.

\subsubsection{Optimised actuator dimensioning}

Although a feasible solution to the design problem introduced in the previous section can be found by trial-anderror, we have implemented an optimisation procedure in order to find an optimal solution. Anthropometric data (NASA, 1978) was used for the verification of the dimensions of the configuration to be incorporated into the exoskeleton structure. As the primary goal of the powered knee joint is to adequately support human knee joint function during treadmill walking, we have used clinical gait analysis (CGA) data (Winter, 1991) for the estimation of the required joint torque and joint range of motion. In the absence of clear guidelines for the actuation system design of powered exoskeletons for gait rehabilitation, these CGA data combined with additional uncertainty margins served as a reasonable first approximation. Experiments with healthy and impaired subjects wearing the powered knee exoskeleton will allow us to improve these design guidelines in view of the development of the complete gait rehabilitation robot. For now, the following design objectives have been taken into account in the design process

- Minimising overdimensioning of the torque-angle characteristic with respect to the required characteristic based on CGA data, which also adds to system safety and compactness.

- Minimising the loads exerted by the PPAM on the structure to obtain a lightweight design.

- Maximising the compactness of the configuration.

The optimal solution meeting these objectives should also satisfy the following set of constraints

- The torque-angle characteristic should exceed the minimal required characteristic.

- Singular configurations may not occur in the range of operation.

- The range of operation should comply with the contraction range of the PPAMs.

- Spatial constraints to avoid obstruction and to limit size.

Thanks to a careful choice of the objectives the optimisation problem can be decomposed into two separate optimisation problems in each parameter set $X_{i}$, defined in Equation 2, and is formulated as follows

Find $X_{i}=\left[l_{0 i}, R_{i}, s_{i}, p_{i}, \epsilon_{0 i}, b_{i}, l_{i}, f_{i}, d_{i}, c_{i}, g_{i}, \alpha_{i}\right]$

To minimise $F_{i}\left(X_{i}\right)=\left[F_{i}^{1}, F_{i}^{2}, \ldots, F_{i}^{n}\right]$

Subject to $C_{i}\left(X_{i}\right)=\left[C_{i}^{1}, C_{i}^{2}, \ldots, C_{i}^{m}\right] \geq 0$.

This is a multi-objective optimisation problem where $F_{i}$ is the vector of $n$ non-linear objective functions and $C_{i}$ is the vector of $m$ non-linear constraint functions. We have used an exhaustive search-based method to find a solution to this optimisation problem. The rationale for this approach is twofold. The discretisation of the search space is justified by the discrete nature of parameters representing dimensions of the mechanical parts. Also, many multi-objective optimisation techniques only provide local optima due to scalarisation (i.e. reformulation of multiple objectives into a single objective), whereas an exhaustive search method does not require prior intervention of the designer to point out preferences between objectives.

In the exhaustive search process, combinatorial candidates are generated and checked for constraint violations. Afterwards, the set of valid candidates is searched for Pareto optimal solutions, i.e. solutions which cannot be improved upon without hurting at least one of the objectives. Because this approach is computationally intensive, we have reduced the search space by reducing the number of parameters, adding boundary constraints and choosing an 


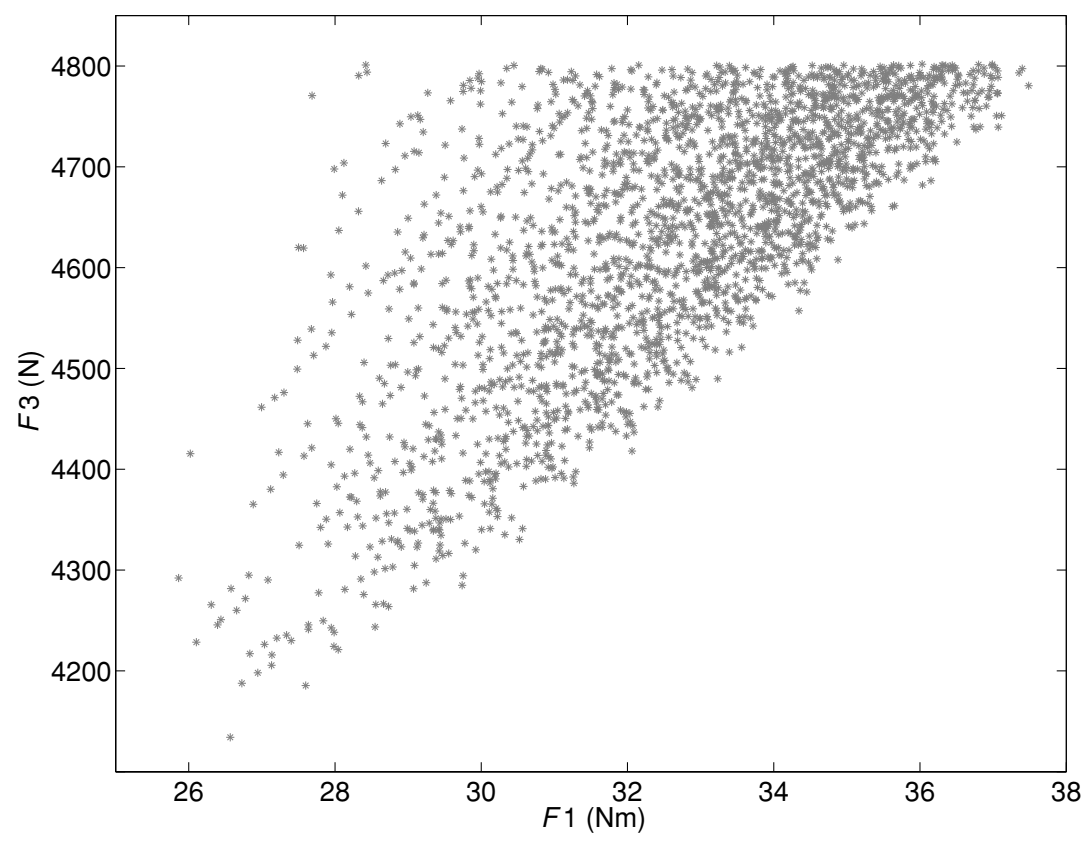

Figure 6. Representation of a full set of candidate solutions in $\left(F_{1}, F_{3}\right)$ axes.

adequate discrete step size. Due to spatial constraints only a relatively small range of PPAM dimensions are retained in the solution space. Therefore $L_{i}, R_{i}, s_{i}, p_{i}$ were assigned a fixed value. Non-dominating parameters $b_{i}$ and $l_{i}$, pointed out by sensitivity analysis, were also kept constant and left out of the parameter set. This left us with 6 remaining parameters $\epsilon_{0 i}, f_{i}, d_{i}, g_{i}, c_{i}$ and $\alpha_{i}$. A full set of candidate solutions, i.e. combinatorial candidates checked for constraint violation, is depicted in Figure 6. Each candidate is represented here by a dot in $\left(F_{1}, F_{3}\right)$ axes. $F_{1}$, the objective function related to minimising torque overdimensioning, is the quadratic mean of the difference between the required maximal torque and the actual maximal torque output. $F_{3}$, the objective function related to minimising the loads acting on the structure, is the maximal actuator force output during operation. Optimal solutions in terms of the represented objective function values are likely to be found in the bottom left corner. Besides the distribution of solutions in the search space, one can gather how much can be gained by optimisation and whether it is justified to search for an optimal solution. $F_{1}$ and $F_{3}$, for instance, drop more than $20 \%$ and $40 \%$ respectively, when, instead of the optimal solution, the worst feasible solution is chosen. So, even in this highly constrained search problem considerable improvement can be achieved.

The code to generate Pareto optimal solutions has been developed in MATLAB, with bottleneck calculations in C code to speed up the analytic synthesis of the four bar linkage mechanism. Figure 7 shows a value path illustration of Pareto optimal solutions for the design problem related to the extensor side of the configuration depicted in Figure 5, with $l_{01}=0.1 \mathrm{~m}, R_{1}=0.0125 \mathrm{~m}, s_{1}=2, p_{1}=1$, $b_{1}=0.06 \mathrm{~m}$ and $l_{1}=0.3 \mathrm{~m}$. The objective function values have been normalised by means of the optimal single objective values for easier interpretation and comparison. Evidently, each single objective optimum, marked with a dashed line, is a Pareto optimum. Knowledge of the full set of Pareto optimal solutions and its distribution helps the decision maker either selecting one single solution or redefining the objective vector in order to change or reduce the set of solutions. Preferences between objectives can be formulated based on this gained knowledge. Possible strategies include transforming objectives into constraints or formulating a single weighed sum objective. In Figure 7 for instance, the optimal solution in terms of a linear combination of objective values with equal weights is marked in bold. The related optimal parameters are $\epsilon_{01}=0.29$, $f_{1}=0.050 \mathrm{~m}, d_{1}=0.063 \mathrm{~m}, g_{1}=0.057 \mathrm{~m}, c_{1}=0.072 \mathrm{~m}$ and $\alpha_{1}=\pi / 3$. Figure 8 shows this particular weighed sum objective function as a function of each parameter, while all other parameters are set equal to the optimal parameter values and held constant. The objective function has been scaled with the objective function value of the optimal solution (indicated by a black dot). From these graphs we learn that the solution space is relatively confined in the vicinity of the optimal solution and that the optimal solution itself lies on the boundary. The complete set of feasible solutions, however, covers a much larger range of parameter values. The sensitivity of the objective function relative to each of the parameters can be found by taking the derivative of 


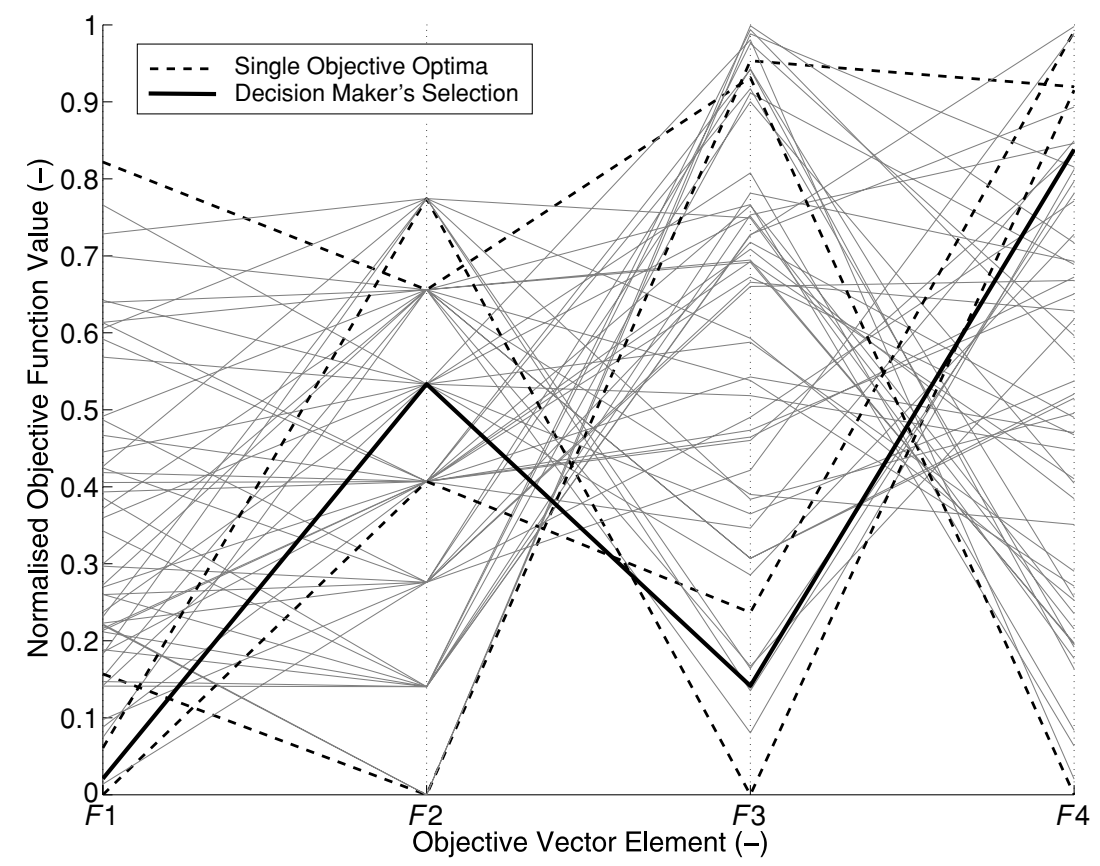

Figure 7. Value path illustration of a Pareto optimal solution set with single objective optima dashed and an example of a decision maker's selection in bold.
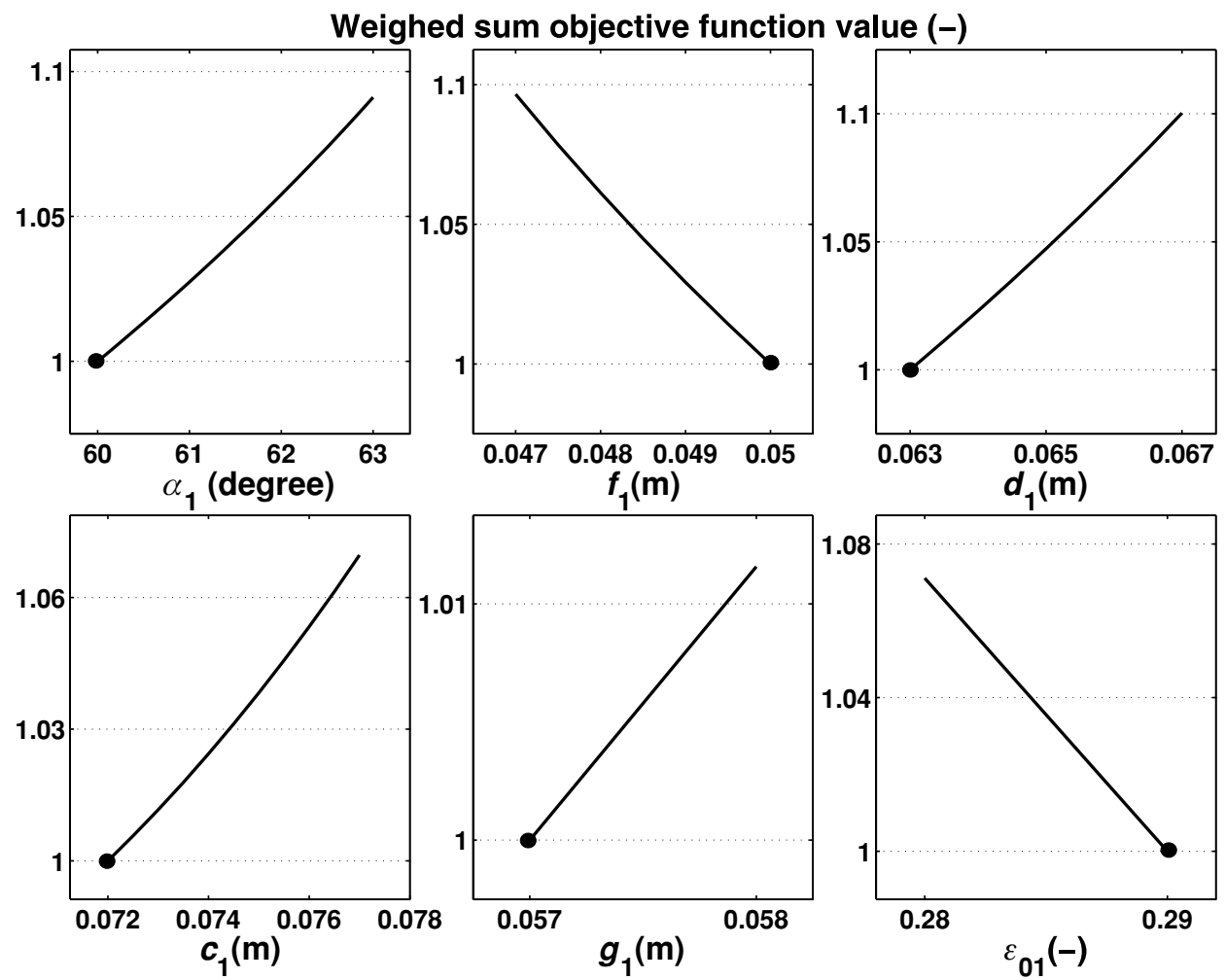

Figure 8. Weighed sum objective function as a function of each parameter, while all other parameters are held constant. The objective function has been scaled with the objective function value of the optimal solution. The optimal solution is indicated by a black dot. 


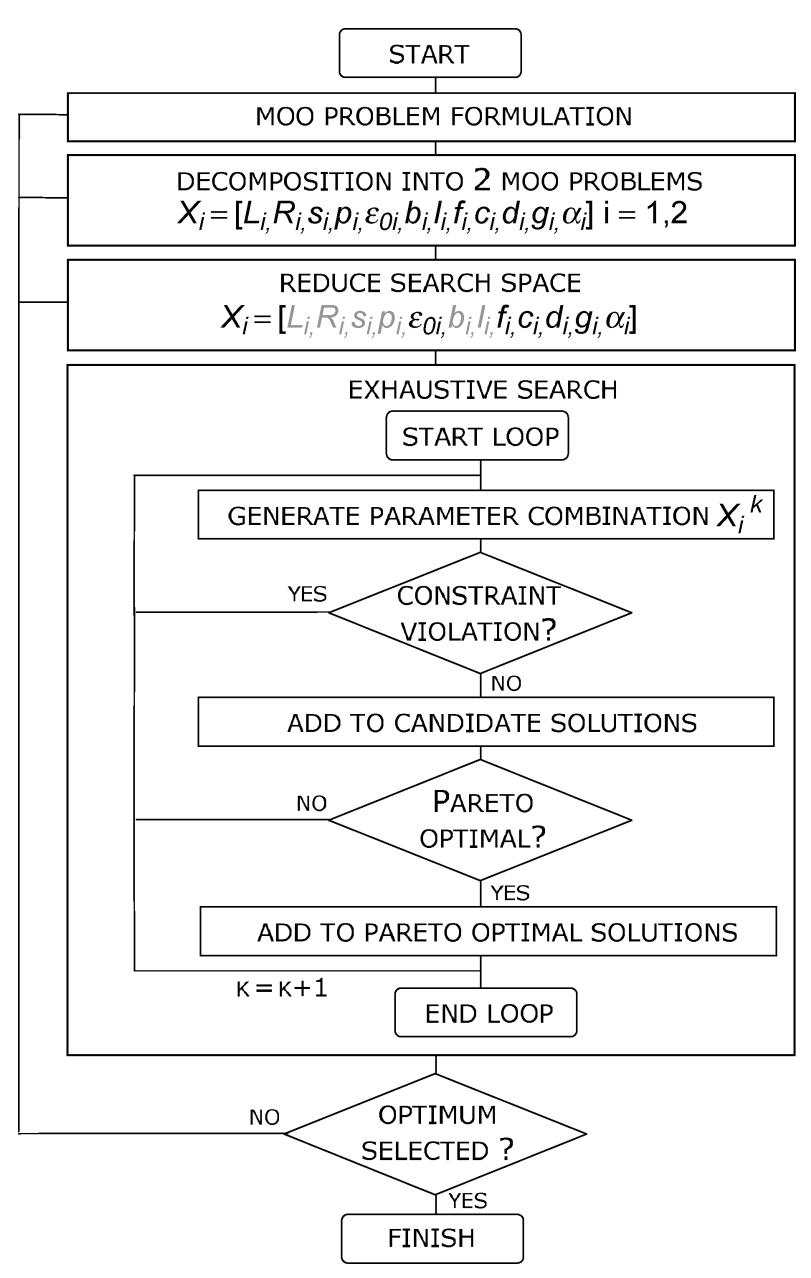

Figure 9. Flow chart of the exhaustive search optimisation procedure.

each of the curves. A $6 \%$ change of parameter $d_{1}$ causes for instance a drop in optimality of $10 \%$. The entire optimisation procedure is summarised in a flow chart depicted in Figure 9.

We have used this code to find an optimal design for the PPAM powered knee joint of the gait rehabilitation exoskeleton. Figure 10 shows the torque-angle characteristic of the exoskeleton's knee joint compared to the minimal required characteristic and to a torque-angle plot for a typical walking cycle of a person with a body weight of $90 \mathrm{~kg}$ (both based on CGA data (Winter 1991)). The bottleneck for the positive torque characteristic is the large extensor torque required after heel strike with the knee slightly bent. In case of small knee joint angles the extensor PPAM is at its highest contraction level, generating its lowest force output. The negative (flexor) torque characteristic is predominantly determined by the large operating range. Due to these bottlenecks and the fact that the torque-angle relationship cannot be arbitrarily shaped, the actuator system is overdimensioned in the range corresponding with low PPAM contraction levels and high pulling forces. We expect the added value of the proposed optimisation procedure to be higher for the design of the complete gait rehabilitation robot, as the latter will benefit from experiencebased guidelines and from the mutual influence of each of the optimised joints.

\section{Control}

\subsection{System requirements}

Since a medical rehabilitation device closely interacts with patients, we consider safety the most important prerequisite. Moreover, due to impaired motor control, muscle weakness and deconditioning, the patient is virtually a slave to the machine during therapy and literally in a vulnerable position. Therefore, safety aspects are to be considered in all stages of design and safety measures should be incorporated on the software level (control) as well as the hardware level. Thanks to the intrinsic compliance of the actuation system deviations from the target position are allowed and passively counteracted without complex feedback control mechanisms, which adds to the safety of the system (Bicchi and Tonietti 2004). Obviously this affects tracking performance, however, our goal is to achieve functional walking without putting too much emphasis on the accuracy of the gait pattern. We believe the compliant behaviour might substantially improve human-robot interaction, especially for patients with spastic hypertonicity of the leg muscles. To this end, a suitable control strategy is needed that does not counteract the intrinsically compliant behaviour of the actuators. Compliance could be adapted online with gait phase and according to the patient's comfort and walking abilities.

Although we aim at a specific target patient group, namely motor incomplete SCI patients, there is a large intersubject variability in terms of, for instance, residual motor control (Maynard et al. 1997). Moreover the principles of motor learning and recovery in robot-assisted neuro-rehabilitation are far from fully understood and therefore difficult to translate into controller design guidelines (Reinkensmeyer et al. 2007). We envisage different control strategies: a trajectory tracking approach with compliance adaptation for patients with poor motor control and a force amplification approach for specific gait support of patients with sufficient voluntary locomotor capability. In (Veneman et al. 2007) a position controlled rehabilitation robot moving the limbs of a passive subject is said to be in 'robot-in-charge' mode, whereas allowing for a subject to move freely without being hindered by the device is called 'patient-in-charge' mode. Intermediate or hybrid control strategies might be appropriate for patients who do not fit in either end of the spectrum.

The exoskeleton prototype will serve as a test setup for the implementation of these control strategies on a single actuated joint. The 'robot-in-charge' or trajectory tracking approach is investigated first, because it allows for the 


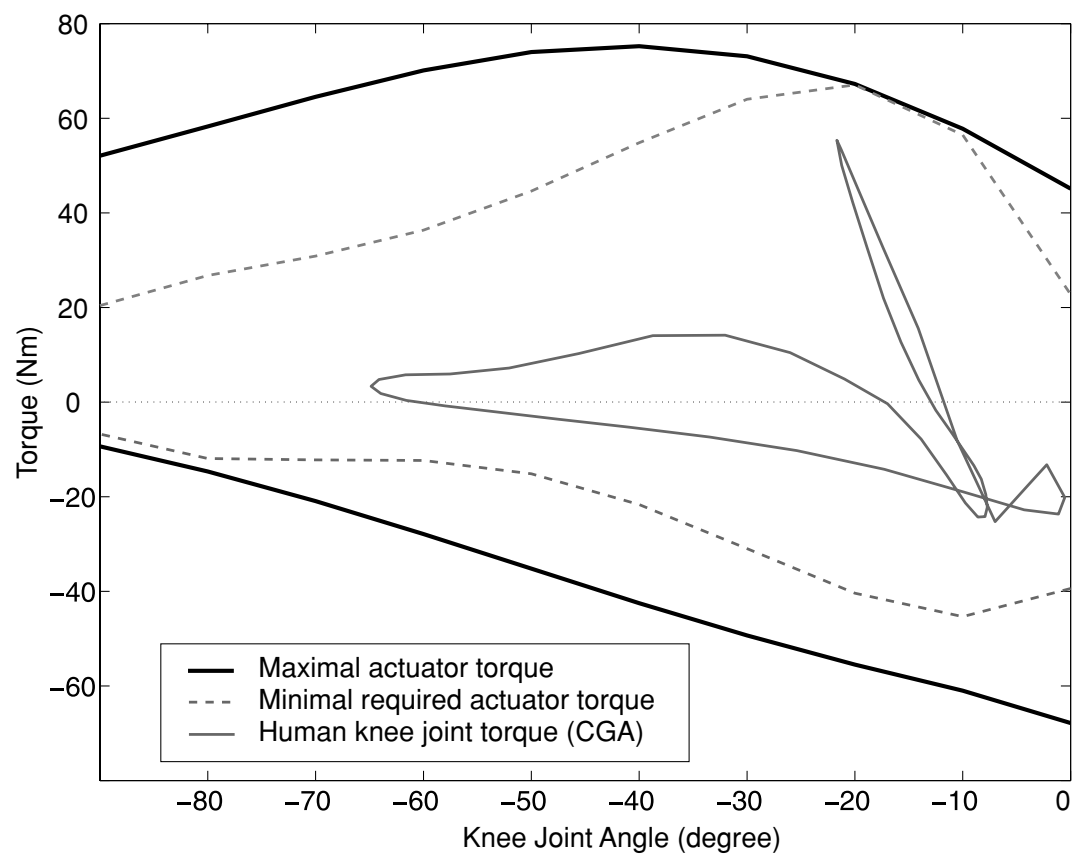

Figure 10. Torque-angle characteristic of the exoskeleton's knee joint compared to the minimal required characteristic and to a torqueangle plot for a typical walking cycle of a person with a body weight of $90 \mathrm{~kg}$ (both based on CGA data (Winter 1991)).

evaluation of system performance in case of maximal power output. In this context we propose PSMC, a new control method introduced by Kikuuwe and Fujimoto (2006). It has been demonstrated experimentally in Kikuuwe and Fujimoto (2006) for a manipulator powered by an electric drive and in Van Damme et al. (2009) for a manipulator powered by pneumatic muscles, that it combines good tracking performance with a safe response to large deviations from the target position. In Van Damme et al. (2009) it was also shown that PSMC considerably improved system safety when compared with PID control. The following section briefly explains the general idea behind PSMC control applied to the gait rehabilitation exoskeleton. For a more detailed description of the control methodology, the reader is referred to Van Damme et al. (2009).

\subsection{Proxy-based sliding mode control}

In PSMC a proxy or virtual object is simulated, that is connected to the robot's end effector by a PID-type virtual coupling. The proxy's position is controlled by an ideal sliding mode controller in order to track the reference position. Unlike sliding mode control PSMC has a continuous output and, therefore, does not suffer from chattering. In Van Damme et al. (2009) a modified form of PSMC is suggested, so that each robot link is controlled separately (joint-space implementation) instead of solely the end effector (task-space implementation).
Now, let us consider a simplified representation in the sagittal plane of a human leg in swing phase attached to the exoskeleton as depicted in Figure 11. Assuming an ideal, rigid connection, we model the combined system as a $3 \mathrm{DOF}$ serial linkage consisting of a hip link fixed to the reference

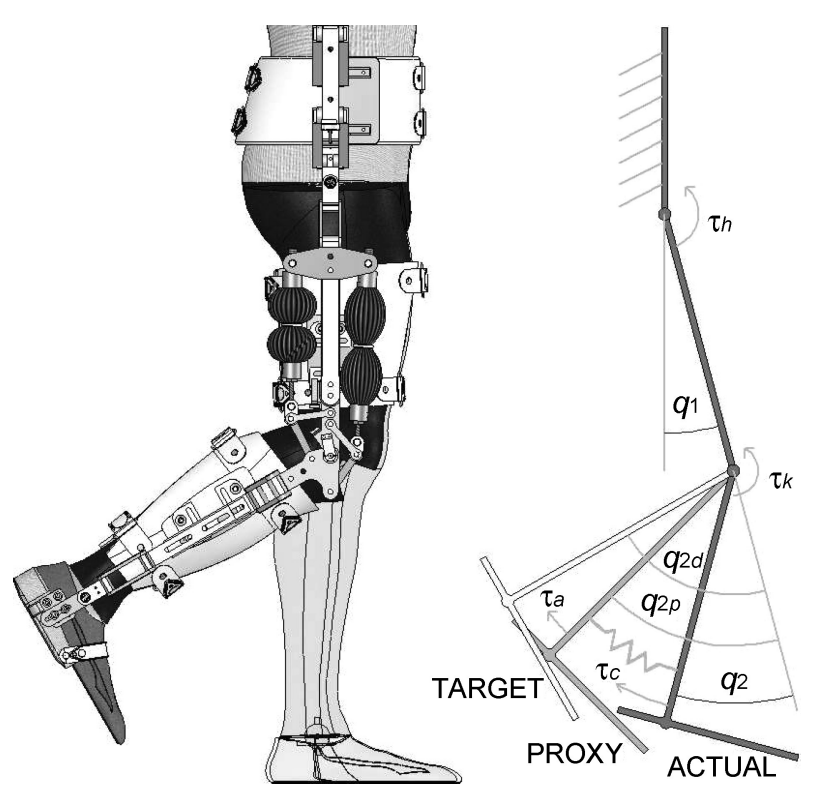

Figure 11. Serial linkage model (right) of the system (left) consisting of the gait rehabilitation exoskeleton and a human leg in swing phase. 
frame and a thigh, shank and foot link. To simplify the model further we lock the ankle joint so that the foot link is perpendicular to the shank link. This leaves us with a 2 DOF system.

Returning to PSMC and applying the control method to the powered knee joint, we distinguish the actual position $q_{2}$ of the lower leg link, the target position $q_{2 d}$ and the position of the proxy $q_{2 p}$, in this case a virtual lower leg link (see Figure 11). The virtual link is attached to the real link by means of a torsional PID-type virtual coupling (parameters $K_{p}, K_{i}$ and $K_{d}$ ) exerting a torque $\tau_{c}$. In PSMC this is the torque we choose to apply to the real link by means of the actuators. On the other hand a virtual torque $\tau_{a}$, the output of a sliding mode controller (sliding mode time constant $\lambda$ ), is applied to the virtual link in order to track the target link. The main advantage of this approach is the separation of 'local' and 'global' dynamics. The local dynamics, i.e. the response to small positional errors, are determined by the virtual coupling (parameters $K_{p}, K_{i}$ and $K_{d}$ ), while the global dynamics (response to large positional errors) are determined by the sliding mode parameter $\lambda$. As a result PSMC combines responsive and accurate tracking during normal operation with smooth, slow and safe recovery from large position errors. The transition between local and global dynamics is determined by a torque magnitude limit $\tau_{\text {lim }}$. As long as the external torque stays below this limit, the controller will drive the proxy to its sliding surface in finite time. Once on the sliding surface, it will exhibit a smooth, exponential convergence (determined by the time constant $\lambda$ ) to the target trajectory. With a properly tuned PID-type virtual coupling, the lower leg link will follow the proxy closely. If an external disturbance (e.g. a torque generated by the patient) acting on the lower leg link exceeds $\tau_{\text {lim }}$, the torque transferred by the virtual coupling to the proxy will pull it off its sliding surface. The lower leg link will be pushed out of position while resisting the disturbance with a torque that equals $\tau_{\mathrm{lim}}$.

\subsection{Simulations}

\subsubsection{Dynamic model}

The primary objective of the simulation study is to assess the control performance of PSMC, before proceeding with the experimental validation on the prototype. The dynamical model of the 2 DOF system, described in Subsection 3.2, is formulated in Equation (6), with $H$ and $C$ the inertia and centrifugal/coriolis matrix, $G$ the gravitational torque vector, $\tau=\left[\tau_{h}, \tau_{k}\right]^{T}$ the vector of joint torques and $q=\left[q_{1}, q_{2}\right]^{T}$ the vector of joint angles. The inertial properties have been estimated by means of CAD software and anthropometric data (NASA, 1978). In Equation (7) the pressure dynamics are modelled by a combination of a first-order system, approximating valve dynamics, and the polytropic law linking angular variations to pressure varia- tions inside the PPAMs (see Equation 5 in subsection 2.2.2). Here $T_{i}$ is a time constant, $p_{i d}$ the desired pressure, $V_{i}(\theta)$ the muscle volume, $P_{\text {atm }}$ the atmospheric pressure, $n$ the polytropic coefficient and $i=1,2$ denoting the extensor PPAM and flexor PPAM, respectively.

$$
\begin{array}{r}
H(q) \ddot{q}+C(q, \dot{q}) \dot{q}+G(q)=\tau \\
\dot{p}_{i}=\frac{p_{i}}{T_{i}}+\frac{p_{i d}}{T_{i}}-n\left(p_{i}+P_{\mathrm{atm}}\right) \frac{1}{V_{i}\left(q_{2}\right)} \frac{d V_{i}\left(q_{2}\right)}{d q_{2}} \dot{q}_{2} .
\end{array}
$$

With the $\Delta p$-approach (Daerden et al. 2001), controller outputs $p_{i}$ are reduced to one single output $\Delta p$, added to or subtracted from the desired mean pressure $p_{m}$. The torque generated by the actuation system (see Equation 3) can thus be reformulated into Equation 8. The PSMC implemented here is based on the modified PSMC formulation (Van Damme et al. 2009) mentioned in the previous section. Besides being a joint space implementation of PSMC the controller directly outputs $\Delta p$ instead of $\tau$. In this way the model-based torque to $\Delta p$ conversion in Equation 8 is bypassed. Consequently, the control parameter $\tau_{\text {lim }}$ becomes $\Delta p_{\text {lim }}$. A gravity and inertia compensation term $\Delta p_{f f}$ is added to the controller output, which results in the control law stated in Equation (9). The reader is referred to Van Damme et al. (2009) for the mathematical formulation of $\Delta p_{\mathrm{psmc}}$ and a detailed description.

$$
\begin{aligned}
\tau_{\text {act }} & =p_{m}\left(m_{1}+m_{2}\right)+\Delta p\left(m_{1}-m_{2}\right) \\
\Delta p & =\Delta p_{f f}+\Delta p_{\text {psmc }}
\end{aligned}
$$

In simulation the virtual patient performs a gait like motion with the leg suspended and attached to the exoskeleton. He is assumed to generate the total required power at the hip joint and virtually no power at the actuated knee joint. The required hip and knee joint trajectories are based on reference trajectories fitted to CGA data (Winter 1991). Each reference trajectory $\theta_{r}\left(\omega_{r}, t\right)$, taking the form of Equation 10 , is shaped into a desired trajectory $\theta_{d}(t)$ by means of an offset $\theta_{0}$, a scale factor $c$ and an angular frequency $\omega$ as in Equation 11. A similar parametrisation has been shown to be effective in covering a broad range of natural walking trajectories (Riener et al. 2005).

$$
\begin{aligned}
\theta_{r}\left(\omega_{r}, t\right) & =\sum_{i=0}^{k}\left(a_{i} \sin \left(i \omega_{r} t\right)+b_{i} \cos \left(i \omega_{r} t\right)\right) \\
\theta_{d}(t) & =\theta_{0}+c \theta_{r}(\omega, t)
\end{aligned}
$$

Since we are mainly interested in the knee joint dynamics at this point, the hip joint kinematics are tracked by a high gain tracking controller. To add to the realism, 


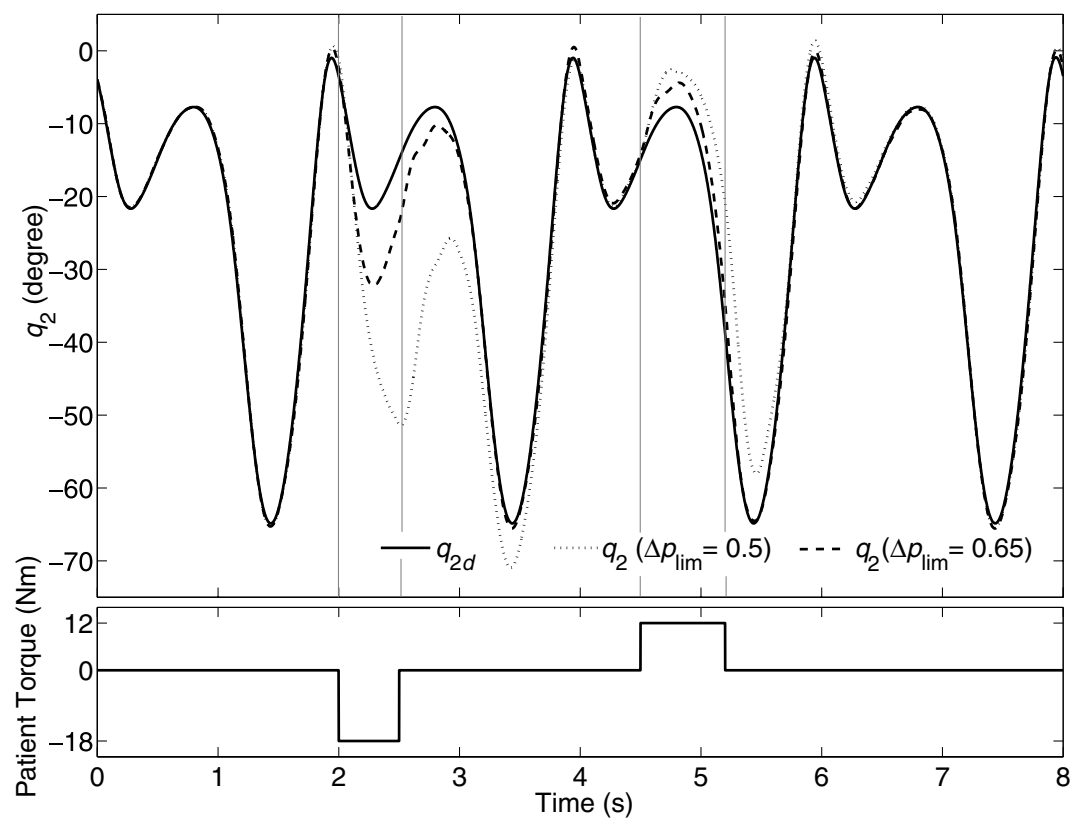

Figure 12. Simulated system response to perturbations by a virtual patient for $\lambda=0.5 \mathrm{~s}$ and different values of control parameter $\Delta p_{\text {lim }}$ (bar).

we have introduced into the controller system parameter uncertainties.

\subsubsection{Simulation results}

By means of the simulation model, implemented in MAT$\mathrm{LAB}$, we have evaluated the performance of the controller in the case of undisturbed trajectory tracking and in the case of perturbations and large deviations from the target position. Figure 12 and 13 show simulation results of the system tracking a gaitlike knee joint trajectory (see Equation 11) with a period of 2 seconds, during which a virtual patient torque is imposed. These results are illustrative for the dual behaviour with respect to undisturbed tracking on the one hand and external disturbances on the other. Depending on the value of control parameter $\Delta p_{\text {lim }}$, a given

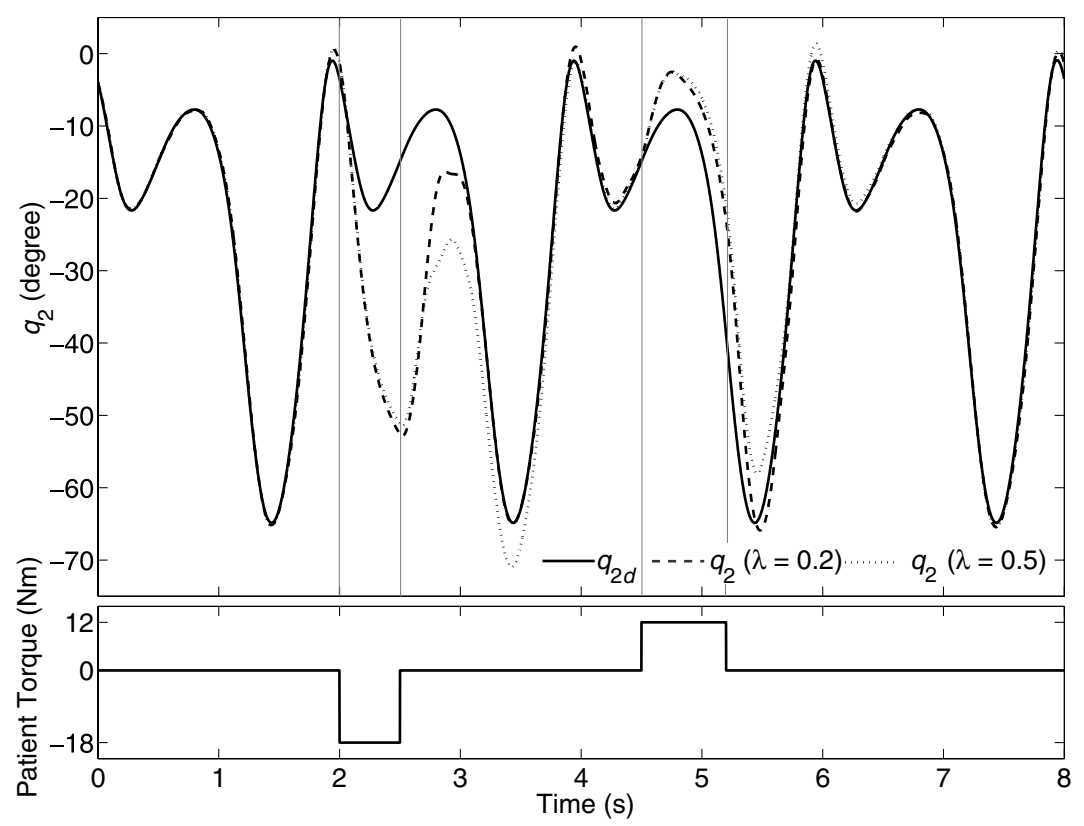

Figure 13. Simulated system response to perturbations by a virtual patient for $\Delta p_{\lim }=0.5$ bar and different values of control parameter $\lambda(\mathrm{s})$. 
disturbance will either cause minor deviations from the target trajectory $\left(\Delta p \leq \Delta p_{\text {lim }}\right)$ or large deviations $(\Delta p \geq$ $\left.\Delta p_{\text {lim }}\right)$ followed by a slow and smooth recovering motion as can be seen in Figure 12. Increasing control parameter $\lambda$ results in a slower response towards the target trajectory as can be seen in Figure 13. The smooth, adjustable recovering motion and adjustable maximal restoring force considerably improve system safety. Both figures also show that in the absence of large perturbations PSMC achieves good tracking performance for this application, even with the large deviations in the system model used by the controller. In view of robot-assisted rehabilitation of gait we consider the observed controller behaviour more compatible with the occurrence of muscle spasms and voluntary muscle activity than what could be expected from conventional feedback controllers. As these simulation results encouraged experimental validation of PSMC, we have tuned and evaluated the PSMC on a simplified test setup with one single DOF and afterwards implemented the controller on the unilateral exoskeleton with powered knee joint.

\subsection{Control hardware}

Data-acquisition is performed by a National Instruments PCI-6229 data-acquisition board at a sampling rate of $500 \mathrm{~Hz}$. Pressure control is done by Kolvenbach KPS 3/400 pressure-regulating valves. Hip and knee joint angles are measured by means of Avagotech AEDEA 3300-TE1 high resolution incremental encoders. Each PPAM is equipped with a gauge pressure sensor.

\section{Experiments}

\subsection{Methods}

A healthy male subject $(1.80 \mathrm{~m}, 80 \mathrm{~kg})$ participated in a series of initial walking experiments in the experimental setup depicted in Figure 14. The setup consists of the gravity balanced exoskeleton and a treadmill.

First, the subject stepped at different treadmill speeds, while the actuators were pressure controlled towards zero gauge pressure. Hip and knee trajectories were recorded during 50 steps. The recorded data has been averaged in order to obtain a single reference gait cycle for each treadmill speed. To this end gait data was divided per gait cycle on the basis of state variables, i.e. knee and hip angles and angular velocities. For now we have only used state space information for offline trajectory generation and not for online synchronisation as described in Aoyagi et al. 2007. A reference phase of gait $\left[\theta_{k}, \theta_{h}, \dot{\theta}_{k}, \dot{\theta}_{h}\right]$, indicating the beginning of a gait cycle, was chosen and each transition to the next gait cycle was calculated by searching the nearest neighbour in state space of that reference state. These gait cycles, having a slightly varying duration, were then scaled in time to a single reference time scale. The period of the reference time scale or mean period was calculated as the inverse of the

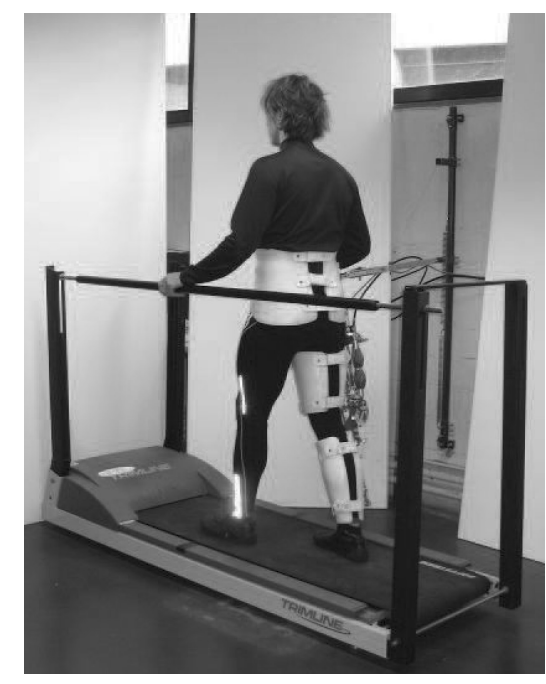

Figure 14. Experimental setup for initial treadmill walking experiments.

principal component of the spectrum of the initial gait data. Finally, the scaled gait cycle data was averaged to retrieve a single reference trajectory for the knee and the hip and the model in Equation 10 (see Subsection 3.3) was fitted to this data.

In the next series of experiments the subject stepped at the same treadmill speeds as in the data recording session, while the PSMC tracked the corresponding reference knee trajectory. Table 1 lists the control parameter values of the implemented PSMC. A short training period was required to familiarise with each walking speed and to synchronise stepping with the device. Hip and knee trajectories as well as muscle gauge pressures were recorded.

\subsection{Experimental results}

Figure 15 and 16 show results of walking experiments with the subject walking at a speed of $0.7 \mathrm{~m} / \mathrm{s}(2.5 \mathrm{kmph})$. This is a common walking speed in robot-assisted treadmill training of SCI patients (Hornby et al. 2005). The averaged reference gait cycle is depicted in Figure 15 against the corresponding recorded gait data of unassisted treadmill walking. In spite of the variability of the gait

Table 1. Tuned PSMC control parameters.

\begin{tabular}{lc}
\hline & PSMC \\
\hline Parameter & Value \\
\hline$K_{p}[$ bar $/ \mathrm{rad}]$ & 3.5 \\
$K_{i}[\mathrm{bar} / \mathrm{rad} . \mathrm{s}]$ & 3.25 \\
$K_{d}[\mathrm{bar} . \mathrm{s} / \mathrm{rad}]$ & 0.25 \\
$\lambda[s]$ & 0.3 \\
$\Delta p_{\lim }[$ bar $]$ & 0.5 \\
\hline
\end{tabular}




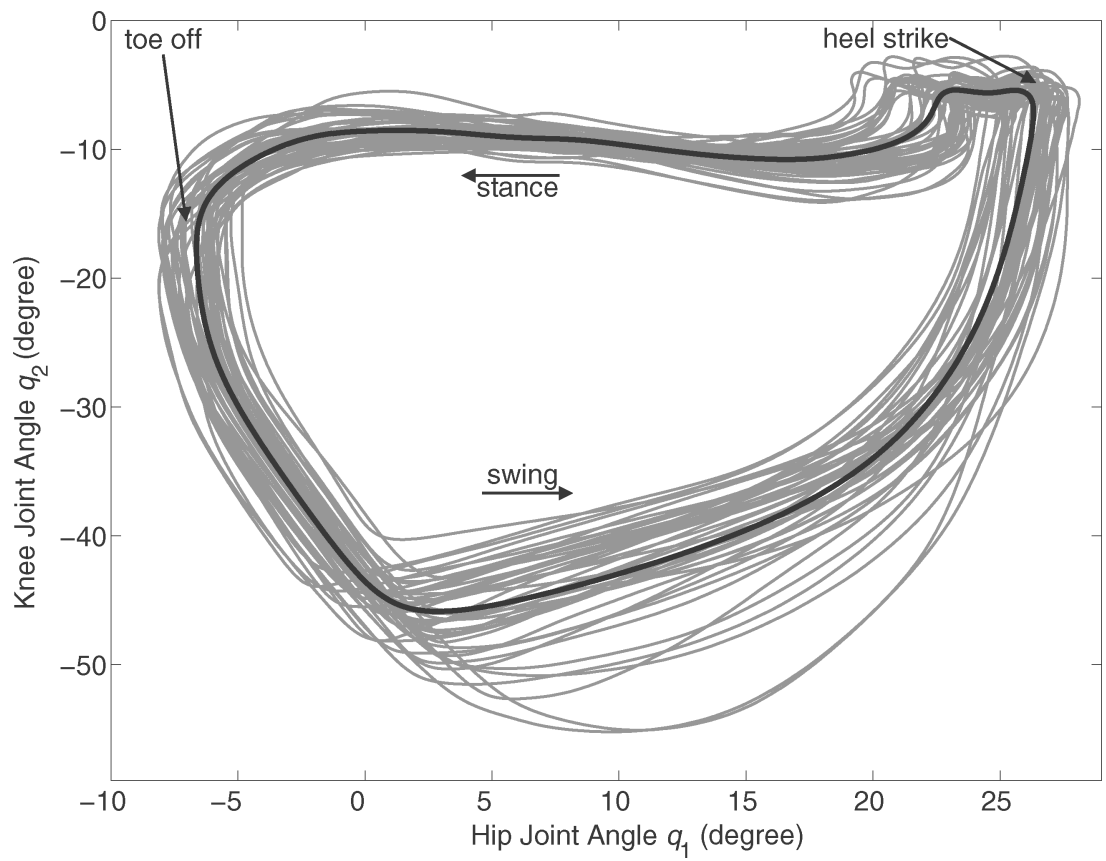

Figure 15. Recorded gait data (grey solid) and averaged reference gait cycle (bold) of a healthy subject wearing the unpowered exoskeleton and walking at a treadmill speed of $0.7 \mathrm{~m} / \mathrm{s}(2.5 \mathrm{kmph})$.

pattern a suitable reference gait cycle has been retrieved to be used as the imposed trajectory during assisted walking. Figure 16 shows the imposed reference gait cycle against the actual gait pattern tracked by the powered exoskeleton.
The largest deviations from the target trajectory occur during swing phase at large knee joint angles (bottom) and at heel strike (top right). Overall variability of the gait pattern is decreased due to the exoskeleton's assistance, but it

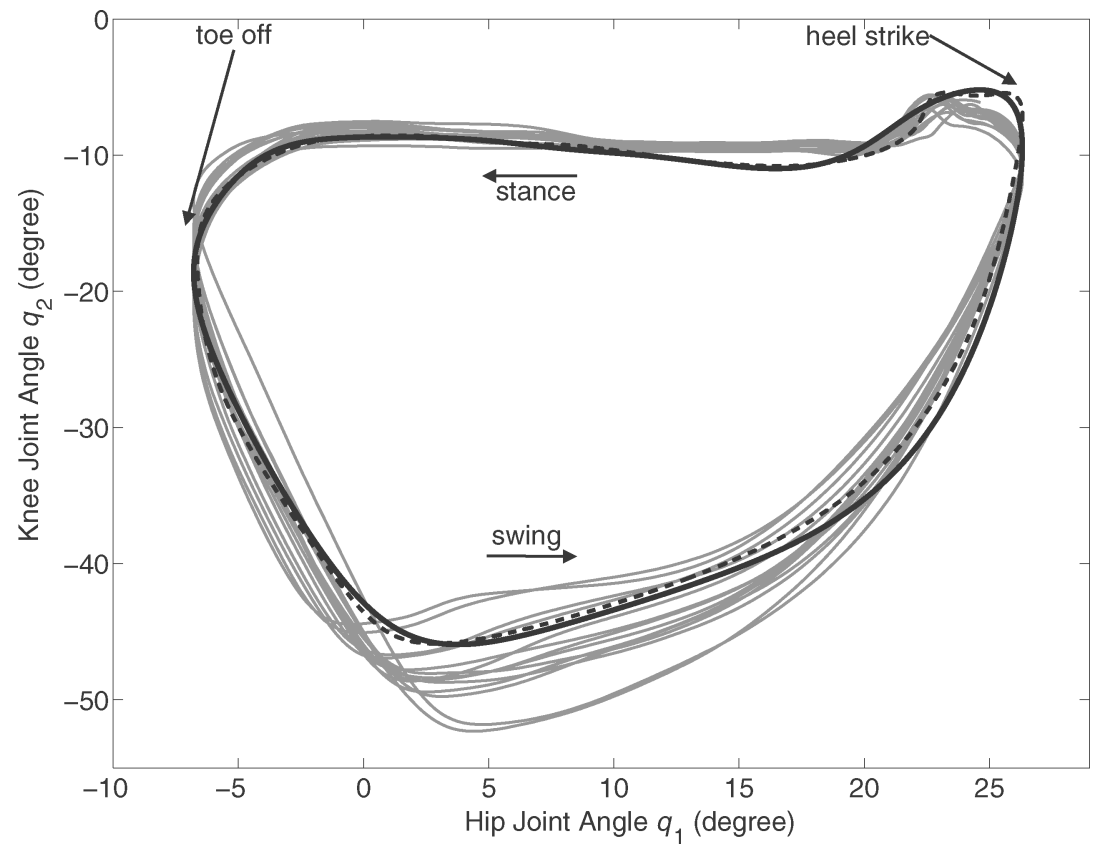

Figure 16. Recorded gait data (grey solid) of a healthy subject wearing the powered exoskeleton and walking at a treadmill speed of $0.7 \mathrm{~m} / \mathrm{s}(2.5 \mathrm{kmph})$, desired gait cycle imposed by the PSMC (dashed) and reference gait cycle (bold). 
is not cancelled out as would be the case for an actuation system with high intrinsic stiffness and high gain feedback. The latter would prevent kinematic errors that are believed to be helpful for motor learning and motivation (Reinkensmeyer et al. 2007). The imposed trajectory felt natural to the subject and more importantly the behaviour of the system was experienced as a gentle guidance without obstructing or heavily counteracting when the subject resisted the imposed motion. Even in case of large deviations, the exoskeleton performed a slow recovery motion towards the target trajectory adding to the perception of comfort and safety. Thus the observed behaviour of the PSMC corresponded well to what was expected on the basis of simulation results.

\section{Conclusion}

In this paper a lower limb exoskeleton prototype with a knee joint powered by pleated pneumatic artificial muscles is presented. The intrinsic compliance, high force output and low weight make the actuator particularly suitable for use in a powered exoskeleton for robot-assisted rehabilitation of gait. The design and control of robotic applications powered by pneumatic artificial muscles are, however, challenging.

The design process was primarily focused on the optimal dimensioning of the actuator configuration. To obtain a compact design, meeting the output requirements and especially the large range of operation, the actuators were connected to the joint by means of a four bar linkage mechanism on either side. A gravity balancing supportive arm with multiple DOF was incorporated into the experimental setup in order to mimic unhindered walking conditions as closely as possible.

For the control of the exoskeleton a 'robot-in-charge' approach was chosen to be investigated first. To preserve the intrinsic safety of the system due to actuator compliance a PSMC was implemented, since it combines good tracking performance with a safe response to large deviations from the target position. The performance of the controller was evaluated in actual gait tracking experiments with a healthy subject wearing the powered exoskeleton while walking on a treadmill. The controller was able to track a reference gait cycle extracted from data recorded during unassisted walking and to realise gentle guidance, even in case of large perturbations imposed by the subject. The user's subjective appreciation of the system in terms of safety and comfort encourage further evaluation of PSMC as a safe 'robot-incharge' control approach to robot-assisted rehabilitation of gait.

\section{Acknowledgements}

This research is funded by a Ph.D grant of the Institute for the Promotion of Innovation through Science and Technology in Flanders (IWT-Vlaanderen). It is supported by a Concerted Research Action, 'ALTACRO, Automated Locomotion Training using an
Actuated Compliant Robotic Orthosis', funded by the Research Council of Vrije Universiteit Brussel - VUB.

\section{References}

Agrawal S, Banala S, Mankala K, Sangwan V, Scholz J, Krishnamoorthy V, Hsu W. 2007. Exoskeletons for gait assistance and training of the motor impaired. Proceedings of the IEEE International Conference on Rehabilitation Robotics. pp. 11081113 .

Aoyagi D, Ichinose W, Harkema S, Reinkensmeyer D, Bobrow J. 2007. A robot and control algorithm that can synchronously assist in naturalistic motion during body-weight-supported gait training following neurologic injury. IEEE Trans. Neural Syst. Rehabil. Eng. 15(3):387-400.

Bicchi A, Tonietti G. 2004. Fast and "soft-arm" tactics [robot arm design]. IEEE Robot Autom Mag. 11(2):22-33.

Colombo G, Jorg M, Schreier RVD. 2000. Treadmill training of paraplegic patients with a robotic orthosis. J Rehabil Res Dev. 37:693-700.

Colombo G, Wirz M, Dietz V. 2001. Driven gait orthosis for improvement of locomotor training in paraplegic patients. Spinal Cord 39:252-253.

Costa N, Caldwell D. 2006. Control of a biomimetic "softactuated" 10dof lower body exoskeleton. Proceedings of the IEEE/RAS-EMBS International Conference on Biomedical Robotics and Biomechatronics. pp. 495-501.

Daerden F, Lefeber D. 2001. The concept and design of pleated pneumatic artificial muscles. Int J Fluid Pow. 2(3):41-50.

Daerden F, Lefeber D. 2002. Pneumatic artificial muscles: actuators for robotics and automation. European J Mech Environ Eng. 47(1):10-21.

Daerden F, Lefeber D, Verrelst B, Van Ham R. 2001. Pleated pneumatic artificial muscles: actuators for automation and robotics. Proceedings of the IEEE/ASME International Conference on Advanced Intelligent Mechatronics. pp. 738-743.

Dollar A, Herr H. 2008. Lower extremity exoskeletons and active orthoses: challenges and state-of-the-art. IEEE Trans Robot. 24(1):144-158.

Ekkelenkamp R, Veltink P, Stramigioli S, van der Kooij H. 2007. Evaluation of a virtual model control for the selective support of gait functions using an exoskeleton. Proceedings of the IEEE 10th International Conference on Rehabilitation Robotics. pp. 693-699.

Emken JL, Harkema SJ, Beres-Jones JA, Ferreira CK, Reinkensmeyer DJ. 2008. Feasibility of manual teach-and-replay and continuous impedance shaping for robotic locomotor training following spinal cord injury. IEEE Trans Biomed Eng. 55(1):322-334.

Ferris D, Sawicki G, Domingo A. 2005. Powered lower limb orthoses for gait rehabilitation. Topics in Spinal Cord Injury Rehabil. 11:34-49.

Hornby T, Zemon D, Campbell D. 2005. Robotic-assisted, bodyweightàsupported treadmill training in individuals following motor incomplete spinal cord injury. Phys Ther. 85(1):52-66.

Jezernik S, Colombo G, Keller T, Frueh H, Morari M. 2003. Robotic orthosis lokomat: a rehabilitation and research tool. Neuromodulation 6 (2):108-115.

Kikuuwe R, Fujimoto H. 2006. Proxy-based sliding mode control for accurate and safe position control. Proceedings of the IEEE International Conference on Robotics and Automation. pp. 2530.

Krebs H, Aisen M, Hogan N. 2000. Increasing productivity and quality of care: robotaided neuro-rehabilitation. J Rehabil Res Dev. 37(6):639-652. 
Maynard F, Bracken M, Creasey G, Ditunno J, Donovan W, Ducker T, Garber S, Marino R, Stover S, Tator C, Waters R, Wilberger J, Young W. 1997. International standards for neurological and functional classification of spinal cord injury. Spinal Cord 35, 266 à 274

NASA, 1978. Anthropometric source book, Vol. I: anthropometry for designers. NASA Scientific and Technical Information Office Hampton, VA, USA.

Rahman T, Ramanathan R, Seliktar R, Harwin W. 1995. A simple technique to passively gravity-balance articulated mechanisms. Trans. of the ASME- J Mech Des. 117(4):655-658.

Reinkensmeyer D, Aoyagi D, Emken J, Galvez J, Ichinose W, Kerdanyan G, Maneekobkunwong S, Minakata K, Nessler J, Weber R, Roy R, de Leon R, Bobrow J, Harkema S, Edgerton V. 2006. Tools for understanding and optimizing robotic gait training. J Rehabil Res Dev. 43(5):657-670.

Reinkensmeyer D, Galvez J, Marchal L, Wolbrecht E, Bobrow J. 2007. Some key problems for robot-assisted movement therapy research: a perspective from the university of california at irvine. Proceedings of the IEEE 10th International Conference on Rehabilitation Robotics. pp. 1009-1015.

Riener R, Lunenburger L, Jezernik S, Anderschitz M, Colombo G, Dietz V. 2005. Patient-cooperative strategies for robot-aided treadmill training: first experimental results. IEEE Trans Neural Sys Rehabil Eng. 13(3):380-394.

Schulte H. 1961. The application of external power in prosthetics and orthotics, Publication 874. National Academy of Sciences - National Research Council, Washington DC. Chapter, The characteristics of the McKibben artificial muscle; pp. 94-115.
Van Damme M, Vanderborght B, Van Ham R, Verrelst B, Daerden F, Lefeber D. 2009. Proxy-based sliding mode control of a planar pneumatic manipulator. International Journal of Robotics Research 28(2): 266-284.

Vanderborght B, Verrelst B, Van Ham R, Van Damme M, Lefeber D, Meira Y Duran B, Beyl P. 2006. Exploiting natural dynamics to reduce energy consumption by controlling the compliance of soft actuators. Int J Robot Res. 25(4):343-358.

Veneman J, Kruidhof R, Hekman E, Ekkelenkamp R, Van Asseldonk E, van der Kooij H. 2007. Design and evaluation of the lopes exoskeleton robot for interactive gait rehabilitation. IEEE Trans Neural Sys Rehabil Eng. 15(3):379-386.

Verrelst B, Van Ham R, Vanderborght B, Lefeber D, Daerden F, Van Damme M. 2006. Second generation pleated pneumatic artificial muscle and its robotic applications. Adv Robot. 20(7):783-805.

Wernig A, Muller S. 1992. Laufband locomotion with body support improved walking in persons with severe spinal cord injuries. Paraplegia 30(4):229-238.

Winter D. 1991. The biomechanics and motor control of human gait: normal, elderly and pathological. 2nd ed. University of Waterloo Press. Waterloo, ON, Canada.

Wirz M, Colombo G, Dietz V. 2001. Long term effects of locomotor training in spinal humans. J Neurol Neurosurg Psychiatry 71:93-96.

Wirz M, Zemon D, Rupp R, Scheel A, Colombo G, Dietz V, Hornby G. 2005. Effectiveness of automated locomotor training in patients with chronic incomplete spinal cord injury: a multicenter trial. Arch Phys Med Rehabil. 86(4):672-680. 

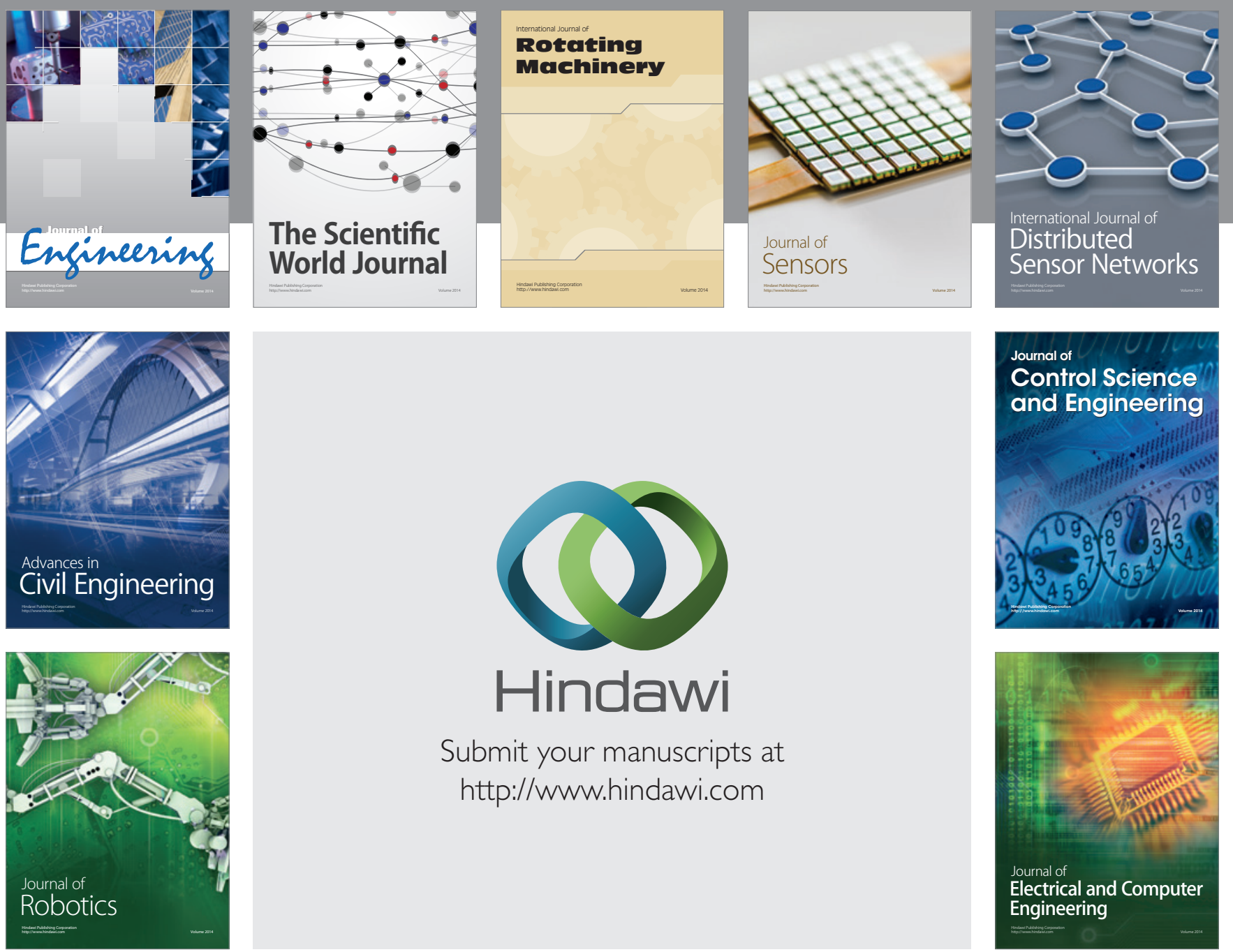

Submit your manuscripts at

http://www.hindawi.com
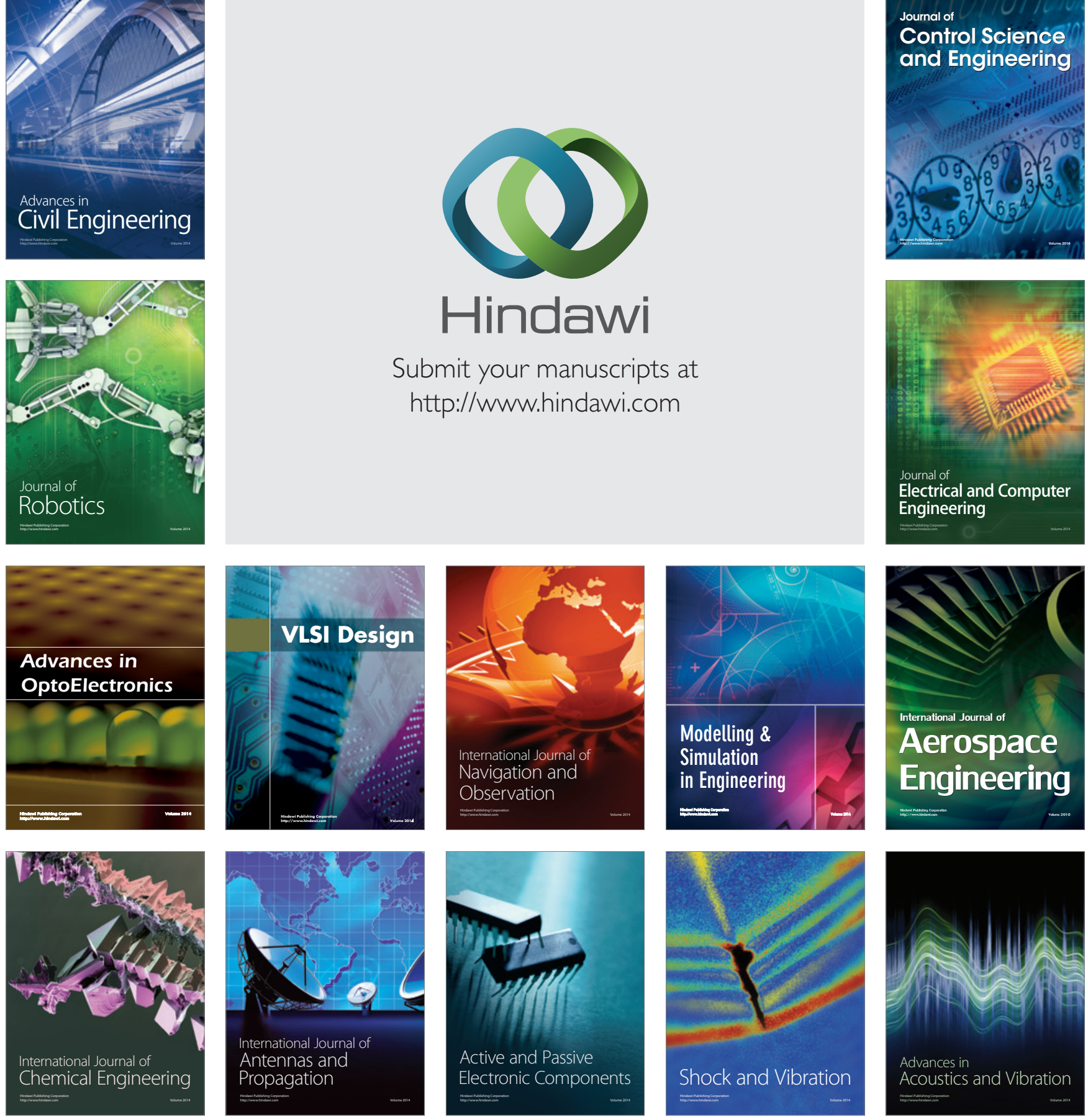